\title{
CSN1 facilitates proliferation and migration of hepatocellular carcinoma cells by upregulating cyclin A2 expression
}

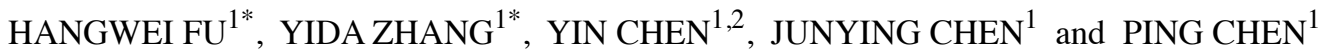 \\ ${ }^{1}$ Department of Hepatobiliary Surgery, Daping Hospital, Army Medical University, Chongqing 400042; \\ ${ }^{2}$ Department of Gynecology and Obstetrics, The 958th Hospital, Southwest Hospital, \\ Army Medical University, Chongqing 400020, P.R. China
}

Received April 8, 2020; Accepted October 14, 2020

DOI: $10.3892 / \mathrm{mmr} .2020 .11684$

\begin{abstract}
Constitutive photomorphogenesis 9 signalosome subunit 1 (CSN1) plays an important role in the ubiquitinproteasome pathway and regulates various cellular processes, such as the cell cycle and DNA repair. The CSN complex consists of eight subunits (CSN1 to CSN8) and regulates the tumorigenesis of a variety of tumor types. However, the exact role of CSN1 in hepatocellular carcinoma (HCC) remains unclear. The present study evaluated the expression and biological effects of CSN1 in HCC tissue samples and cell lines. CSN1 was significantly overexpressed in HCC tissue and cell lines, compared with their normal counterparts. In patients with HCC, elevated CSN1 levels correlated with tumor size, tumor metastasis and tumor stage. Loss-of-function assays indicated that CSN1 knockdown inhibited the proliferation and migration HCC cells. In addition, CSN1 promoted the expression of cyclin A2 in a ubiquitination-independent manner. Lastly, xenograft experiments indicated that CSN1 promoted HCC tumor growth in vivo. The present study suggested that CSN1 inhibition could represent a potential approach for the prevention of HCC progression and metastasis.
\end{abstract}

Correspondence to: Dr Ping Chen or Dr Junying Chen, Department of Hepatobiliary Surgery, Daping Hospital, Army Medical University, 10 Changjiangzhilu Daping, Yuzhong, Chongqing 400042, P.R. China

E-mail: chenpingsyd@126.com

E-mail: junyingchencq@163.com

${ }^{*}$ Contributed equally

Abbreviations: AFP, $\alpha$-fetoprotein; CSN1, constitutive photo morphogenesis 9 signalosome subunit 1; HCC, hepatocellular carcinoma; BCLC, Barcelona Clinic Liver Cancer; HBsAg, hepatitis B surface antigen

Key words: CSN1, HCC, cyclin A2, cell proliferation

\section{Introduction}

Hepatocellular carcinoma (HCC) has the sixth highest incidence among all malignant tumors worldwide and the fourth highest mortality rate worldwide (1). More than half of all patients with HCC worldwide reside in China (2). In recent years, the incidence of liver cancer has been on the rise, yet $<20 \%$ of patients receive timely radical surgical resection, due to the high degree of malignancy, early metastasis and high invasion potential of this tumor type. These properties are often associated with the complex biochemical regulatory behavior of HCC cells, such as excessive cell proliferation $(3,4)$. Although HCC treatment methods and efficiency have improved the 5-year survival rate, several methods such as radical resection, transcatheter arterial chemoembolization, sorafenib and chemoradiotherapy still have their respective limits (5). Radical hepatectomy is considered the first choice for the treatment of early HCC at an early stage (Barcelona Clinic Liver Cancer stage 0 or A). For these patients, resection is associated with survival $>60 \%$ at 5 years, with low postoperative mortality $(<3 \%)$, and as many as $70 \%$ of these patients experience tumor recurrence at 5 years (6). Based on HCC pathogenesis, in addition to a number of correlative studies, immunotherapy represents a potential therapeutic option (7). Therefore, investigating the mechanism underlying the onset of HCC and finding a potential therapeutic target is of significance for improving the diagnosis and treatment of HCC.

Constitutive photomorphogenesis 9 signalosome (COP9) is a protein complex composed of eight subunits (CSN1 to CSN8) that was initially discovered in plants as an inhibitor of photomorphogenesis (8-12). Mammalian COP9 is involved in a variety of biological processes, including cell cycle control, signal transduction, transcriptional activation and tumorigenesis (13-15). CSN subunits have a conserved structure; for example, the Mpr1-Pad1-N-terminal (MPN) domain in CSN5 contains a metalloproteinase motif, which has catalytic activity and is involved in the regulation of protein demethylation (16-18). MPN in CSN6 may serve as a structural scaffold or play a regulatory function $(16,19,20)$. Previous studies have confirmed that CSN inhibits the ubiquitin-dependent degradation of numerous tumor-related proteins (21-23), such as P27, P53, E3 ubiquitin-protein ligase Mdm2, Smad7, Runt-related 
transcription factor 3, DNA-binding protein inhibitor ID-1, S-phase kinase-associated protein 2 and hypoxia-inducible factor 1 (14), indicating that CSN plays an important role in tumorigenesis. In particular, CSN5 or CSN6 overexpression has been detected in numerous types of cancer, such as myeloma, lung cancer and breast cancer $(24,25)$. In HCC, CSN6 overexpression promotes epithelial-mesenchymal transition and predicts poor prognosis (26). Inhibition of CSN3 expression induced growth arrest and apoptosis of HCC cells (27). In our preliminary study, by analyzing the transcriptome data of patients within The Cancer Genome Atlas, it was found that CSN1 expression was increased in patients with HCC compared with normal controls. However, the role and molecular mechanism of CSN1 in HCC remains to be elucidated.

Cyclin A2 is a highly conserved cell cycle protein encoded by the CCNA2 gene. Cyclin A2, combined with cyclin-dependent kinase 1 (CDK1) and CDK2, controls cell cycle progression and promotes mitosis (28) and serves a potential role in liver tumorigenesis (29). Cyclin A2 has been recognized as a marker for several types of cancer diagnosis and prognosis (30), such as lung carcinoma, breast cancer, hepatocellular carcinoma and colorectal cancer. Moreover, the expression of cyclin A2 is increased in HCC (31), indicating that this protein may play an important role in the development of $\mathrm{HCC}$ and represent a potential therapeutic target.

In the present study, CSN1 was upregulated in HCC tissue samples, compared with healthy adjacent tissue. In addition, high levels of CSN1 indicated poor prognosis in patients with HCC. CSN1 knockdown inhibited proliferation and migration and induced apoptosis and cell cycle arrest in HCC cells. Moreover, xenograft tumor experiments demonstrated that CSN1 promoted HCC in vivo. Mechanistically, cyclin A2 may be a downstream effector of CSN1.

\section{Materials and methods}

Patients and sample collection. Primary HCC tissue samples were obtained for retrospective analysis from 117 patients at Daping Hospital, Army Medical University, between January 2013 and December 2014. Matched, adjacent, non-tumor tissue samples resected 1-2 $\mathrm{cm}$ from the malignant tumor were obtained from 59 of these patients. Written informed consent was obtained from all participants. All specimens obtained during surgery were assembled into a tissue microarray (TMA) for further analysis. The identification of tumor tissues and adjacent normal tissues was confirmed by pathologists. The present study was approved by the Institutional Research Ethics Committee of Daping Hospital, Army Medical University.

Cell culture and transfection. Human $\mathrm{HCC}$ cell lines (MHCC-97H, MHCC-LM3 and Hep3B) were purchased from the Cell Bank of Academia Sinica. The immortalized liver cell line MIHA was a kind gift from Dr Deng Huang. The cells were cultured in DMEM (HyClone; Cytiva) supplemented with $10 \%$ FBS (Shanghai ExCell Biology, Inc.) at $37^{\circ} \mathrm{C}$ with $5 \% \mathrm{CO}_{2}$.

Human CSN1 and cyclin A2 small interfering (si) RNA molecules were synthesized by Shanghai GenePharma Co.,
Ltd. The siRNA sequences were as follows: i) si-CSN1-1, 5'-CUGCCGGUUCAGGUGUUATT-3'; ii) si-CSN1-2,5'-GAA CCUUUAACGUGGACAUTT-3'; iii) si-CSN1-3, 5'-GAGACA UCAUCUUCAAAUUTT-3'; iv) si-cyclin A2, 5'-CAACCC ACCAGAGACACUAAATT-3'; and v) si-negative control (NC), 5'-UUCUCCGAACGUGUCACGUTT-3'. Human CSN1 overexpression (OE) plasmid and its corresponding empty vector (pReceiver-M02) were purchased from GeneCopoeia, Inc. Plasmids $(2 \mu \mathrm{g})$ or siRNA $(50 \mathrm{nM})$ were transfected using Lipofectamine ${ }^{\circledR} 2000$ transfection reagent (Invitrogen; Thermo Fisher Scientific, Inc.) according to the manufacturer's instructions. Subsequent cell experiments were conducted after transfection for $48 \mathrm{~h}$.

The $293 \mathrm{~T}$ cells were inoculated in $10-\mathrm{cm}$ culture plates at a density of $5 \times 10^{6}$ cells in $15 \mathrm{ml}$ and allowed to reach $70-80 \%$ confluence the day prior to infection. The lentiviral plasmid GV $(20 \mu \mathrm{g})$, and packaging plasmids pHelper $1.0(15 \mu \mathrm{g})$ and pHelper $2.0(10 \mu \mathrm{g})$ (Shanghai GeneChem Co., Ltd.) were transfected into 293T cells using Genechem Transfection Reagent (Shanghai GeneChem Co., Ltd.) for $6 \mathrm{~h}$ at $37^{\circ} \mathrm{C}$ in $5 \% \mathrm{CO}_{2}$, according to the manufacturer's protocol. Following 48-72 h, supernatants containing lentiviral particles were harvested and filtered through a $0.45-\mu \mathrm{m}$ filter (EMD Millipore) to remove cell debris. The supernatants were concentrated by ultracentrifugation at $25,000 \times \mathrm{rpm}$ at $4^{\circ} \mathrm{C}$ for $2 \mathrm{~h}$, and the lentiviral particle pellet was resuspended in $100 \%$ FBS and stored at $-80^{\circ} \mathrm{C}$. The viral titers of concentrated lentiviral particles were measured by infecting $293 \mathrm{~T}$ cells seeded at a density of $4 \times 10^{4}$ cells/well in a 96-well plate with viral serial dilutions. Green fluorescent protein (GFP) expression was detected 4 days later under a fluorescence microscope and the viral titer was calculated using the following equation: Viral titer $(\mathrm{Tu} / \mu \mathrm{l})=\left(\% \mathrm{GFP}^{+}\right.$cells $\mathrm{x}$ number of cells transduced $) /$ virus volume.

For lentiviral transduction, MHCC-LM3 were seeded at $1 \times 10^{5}$ cells $/ \mathrm{ml}$ in $24-w e l l$ plates, and the concentrated lentivirus was added at $\mathrm{MOI}=20$ at $37^{\circ} \mathrm{C}$ and $5 \% \mathrm{CO}_{2}$. Seven days after transfection, $1 \mu \mathrm{g} / \mathrm{ml}$ puromycin (cat. no. ST551; Beyotime Institute of Biotechnology) was added into the cell culture medium to select the transduced cells.

Western blot analysis. Total protein was extracted from cells using RIPA lysis buffer (Beyotime Institute of Biotechnology), according to the manufacturer's protocol. Total protein was quantified using a BCA Protein Assay kit (cat. no. P0012; Beyotime Institute of Biotechnology) and equivalent amount of $20 \mu \mathrm{g}$ protein samples were separated with a $4-12 \%$ Bis-Tris gel (Beyotime Institute of Biotechnology) and transferred onto a PVDF membrane. The membrane was blocked with 5\% non-fat milk in TBS-Tween-20 for $1 \mathrm{~h}$ at room temperature, incubated with the indicated primary antibodies overnight at $4{ }^{\circ} \mathrm{C}$, and then with horseradish peroxidase-conjugated secondary antibodies (1:5,000; ProteinTech Group, Inc.) at room temperature for $1 \mathrm{~h}$. The primary antibodies used were specific for CSN1 (1:2,000; cat. no. 11709-1-AP; ProteinTech Group, Inc.), cyclin A2 (1:2,000, cat. no. GTX103042; GeneTex, Inc.), CDK-2 (1:2,000; cat. no. 10122-1-AP; ProteinTech Group, Inc.), CDK-4 (1:2,000; cat. no. 11026-1-AP; ProteinTech Group, Inc.), phospho (p)-CDK4 (1:2,000, cat. no. GTX00778; GeneTex, Inc.) or GAPDH (1:5,000; cat. no. 10494-1-AP; ProteinTech 
Group, Inc.). HRP-conjugated Affinipure goat anti-mouse IgG (H+L) (1:5,000; cat. no. SA00001-1; ProteinTech Group, Inc.) and HRP-conjugated AffiniPure goat anti-rabbit $\operatorname{IgG}(\mathrm{H}+\mathrm{L})$ (1:5,000; cat. no. SA00001-2; ProteinTech Group, Inc.) were used as secondary antibodies. Protein signals were detected by enhanced chemiluminescence (cat. no. KGP1121; Nanjing KeyGen Biotech Co., Ltd.). Data were semi-quantified using ImageJ software (v1.48; National institutes of Health). GAPDH was used as internal control.

Immunohistochemistry for TMA. TMAs were constructed from formalin-fixed, paraffin-embedded tissue blocks stored at the Department of Pathology, Daping Hospital. All specimens were previously fixed in $10 \%$ neutral formalin solution at room temperature for $12 \mathrm{~h}$ before being paraffin-embedded and cut to $8-\mu \mathrm{m}$ thick sections. Following incubation at $65^{\circ} \mathrm{C}$ for $1 \mathrm{~h}$, TMA slides were dewaxed with dimethylbenzene and then rehydrated with graded alcohol and distilled water. For antigen retrieval, TMA slides were incubated in a microwave oven heated at $95^{\circ} \mathrm{C}$ with $10 \mathrm{mM}$ citrate buffer ( $\mathrm{pH}$ 6.0). Endogenous peroxidases were quenched with $3 \% \mathrm{H}_{2} \mathrm{O}_{2}$ for $20 \mathrm{~min}$. The slides were treated with normal goat serum (cat. no. $\mathrm{C} 0265$; Beyotime Institute of Biotechnology) to inhibit non-specific staining at room temperature for $15 \mathrm{~min}$. Subsequently, the sections were incubated with anti-CSN1 antibody (1:50; cat. no. 11709-1-AP; ProteinTech Group, Inc.) overnight at $4^{\circ} \mathrm{C}$ and then incubated with goat anti-rabbit antibody (1:500; cat. no. C0265; Beyotime Institute of Biotechnology) for $1 \mathrm{~h}$. Diaminobenzidine (OriGene Technologies, Inc.) was used to produce a brown staining. Once hematoxylin counterstaining and dehydration were completed, the sections were sealed. Immunohistochemical staining of the TMA was assessed by light microscopy. Three random fields of view of the IHC chips were captured. The expression levels of CSN1 were quantified by counting mean gray value with ImageJ (version 1.48; National Institutes of Health). A value higher than the mean was defined as high expression, while a value equal to or lower than the mean was categorized as low expression in tumors.

Cell proliferation assay. HCC cells were seeded into 96-well plates at a density of $5 \times 10^{3}$ cells/well and transfected with the indicated siRNA or plasmids. At $48 \mathrm{~h}$ after transfection, cell proliferation was determined in vitro using the EdU DNA Proliferation Detection kit (Nanjing KeyGen Biotech Co., Ltd.) according to the manufacturer's instructions.

Transwell cell migration and wound healing assays. Transwell chambers (BD Biosciences) with a pore size of $8 \mu \mathrm{m}$ were used to determine cell migration. MHCC-97H or MHCC-LM3 cells $\left(2 \times 10^{4}\right)$ were seeded in the upper chamber in serum-free DMEM (HyClone; Cytiva). Medium containing 10\% FBS was added to the lower chamber. Following incubation at $37^{\circ} \mathrm{C}$ for $24 \mathrm{~h}$, the cells were fixed in methanol and stained with leucocrystal violet at room temperature for $10 \mathrm{~min}$. Cells in the upper chamber were removed, and the number of cells that migrated across the membrane was determined by counting the number of leucocrystal violet-stained cells. Stained cells were examined under a light microscope and quantified by counting all the cells in the field of view. At least three random microscopic fields were captured.
MHCC-97H or MHCC-LM3 cells ( $3 \times 10^{5}$ cells/well) were seeded and transfected as aforementioned in 6-well plates. The cells were then scratched with a $10-\mu 1$ pipette tip along the center of the plate when the cells reached $\sim 95 \%$ confluence. The HCC cells were incubated in serum-free medium. The distances between the cells bordering the wound were measured and analyzed under a light microscope at 0, 24 and $48 \mathrm{~h}$.

Cell cycle and cell apoptosis analysis. For cell cycle and cell apoptosis analysis, MHCC-97H or MHCC-LM3 cells were seeded at a density of $3 \times 10^{5}$ cells/well in a 6 -well plate and transfected with the indicated constructs. At $48 \mathrm{~h}$ after transfection, the cells were harvested by centrifugation at $1,000 \mathrm{x} \mathrm{g}$ for $5 \mathrm{~min}$ and fixed in $70 \%$ ethanol overnight at $4{ }^{\circ} \mathrm{C}$. The cells were then analyzed using DNA Content Quantitation Assay (Cell Cycle) (cat. no. CA1510-50T; Beijing Solarbio Science \& Technology Co., Ltd.) according the manufacturer's protocol.

The apoptosis rate of HCC cells was analyzed using an Annexin V-FITC/PI apoptosis detection kit (cat.no. CA1020-50T; Beijing Solarbio Science \& Technology Co., Ltd.) following the manufacturer's instructions. In brief, $5 \times 10^{5}$ cells were harvested by centrifugation at $1,000 \times \mathrm{g}$ for $5 \mathrm{~min}$ and resuspended in $200 \mu \mathrm{l}$ binding buffer, followed by a 15-min incubation with $5 \mu \mathrm{l}$ Annexin V-FITC and $5 \mu \mathrm{l}$ propidium iodide in the dark at $37^{\circ} \mathrm{C}$. Data were acquired using a FACSVerse flow cytometer (BD Biosciences) and analyzed using FlowJo software (10.0.7; FlowJo LLC). Annexin $\mathrm{V}^{+} \mathrm{PI}$ cells were identified as early apoptotic cells, whereas Annexin $\mathrm{V}^{+} \mathrm{PI}^{+}$cells were considered late apoptotic cells.

Reverse transcription-quantitative PCR (RT-qPCR). Total RNA was isolated from cells using the TRIpure total RNA extraction kit (cat. no. RP1001; BioTeke Corporation). RT was performed using the PrimeScript ${ }^{\mathrm{TM}}$ RT Reagent kit, and qPCR was performed using SYBR Premix Ex Taq II (both from Takara Bio, Inc.) according to the manufacturer's instructions. RT was carried out at $37^{\circ} \mathrm{C}$ for 15 min then $85^{\circ} \mathrm{C}$ for $15 \mathrm{sec}$. qPCR was performed at $95^{\circ} \mathrm{C}$ for $30 \mathrm{sec}$, followed by 40 cycles of $5 \mathrm{sec}$ at $95^{\circ} \mathrm{C}$ and $30 \mathrm{sec}$ at $60^{\circ} \mathrm{C}$. GAPDH was used as reference gene. The following primer pairs were used: GAPDH forward, 5'-ACTCCTCCACCTTTGACGC-3' and reverse, 5'-GCTGTAGCCAAATTCGTTGTC-3'; cyclin A2 forward, 5'-CCAGGAGAATATCAACCCGGA-3' and reverse, 5'-GTG CAACCCGTCTCGTC-3' The relative expression levels of each target were quantified using the $2^{-\Delta \Delta \mathrm{Cq}}$ method (32).

Protein half-life assay. To examine the half-life of cyclin A2 protein, cycloheximide pulse-chase experiments were performed as previously described (33). MHCC-LM3 cells were seeded at $3 \times 10^{5}$ cells/well in 6 -well plates. Twenty-four hours later, the cells were treated with $75 \mu \mathrm{g} / \mathrm{ml}$ cycloheximide (CHX; Sigma-Aldrich; Merck KGaA) to block the endogenous protein synthesis for $0,1,2,4,6$ and $8 \mathrm{~h}$, and were subsequently lysed for western blotting. The bands were scanned, and the image semi-quantified using ImageJ software (v1.48; National institutes of Health). The relative concentration at $0 \mathrm{~h}$ was defined as $100 \%$. The fraction of remaining cyclin A2 at each indicated time point was normalized by comparing the relative concentration with that at time $0 \mathrm{~h}$. 
A

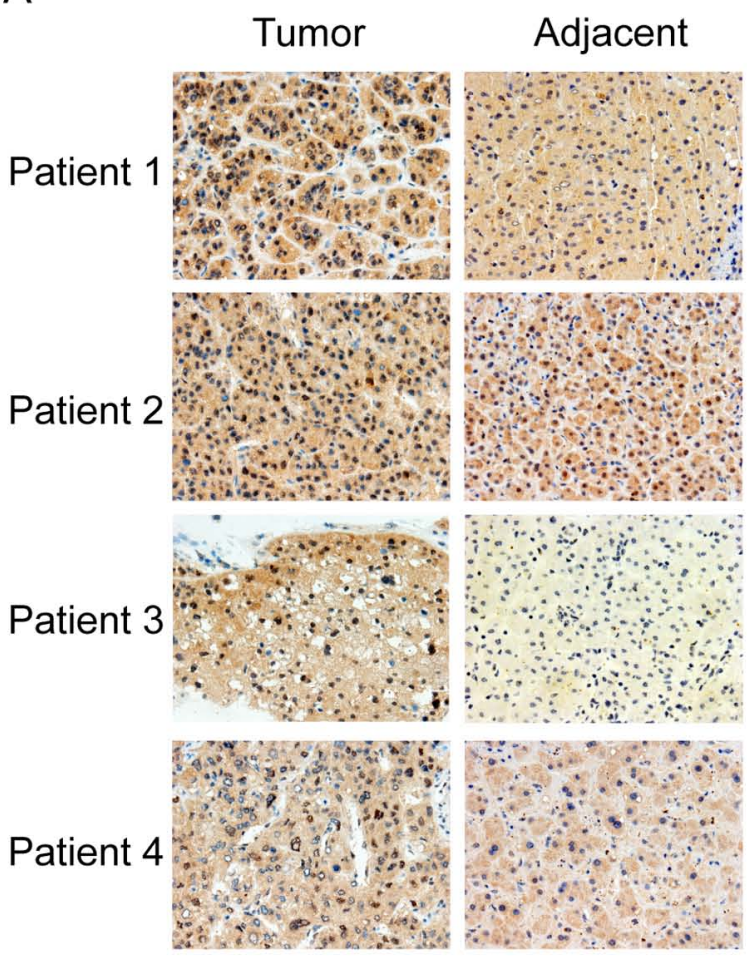

B

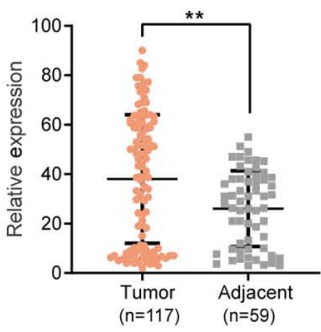

$\mathrm{D}$

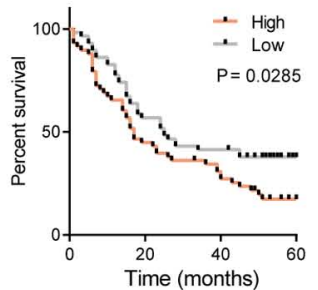

F

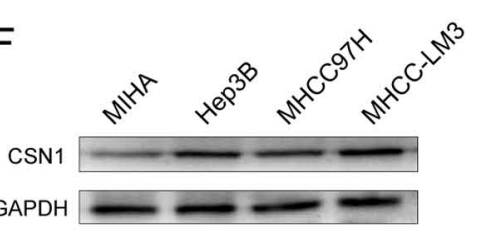

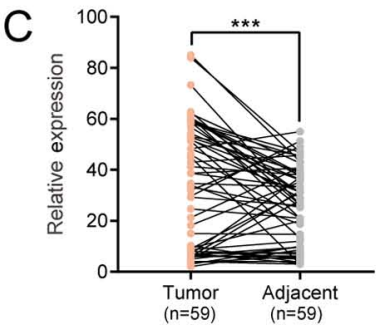

E
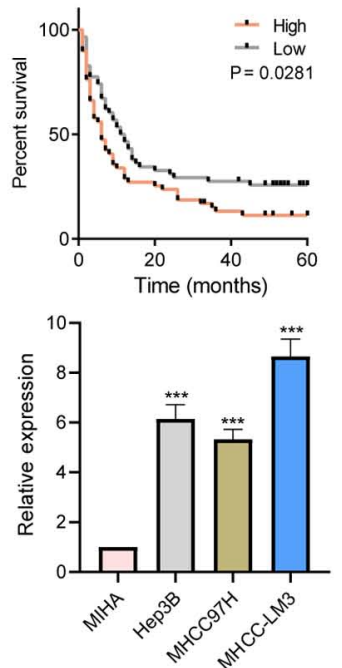

Figure 1. Relative CSN1 expression in HCC and its clinical significance. (A) Tissue microarray immunohistochemical staining. Magnification, x200. (B) Relative expression of CSN1 in HCC tissue ( $\mathrm{n}=117)$ and paracancerous tissue samples $(\mathrm{n}=59)$. (C) CSN1 expression levels in cancer and adjacent tissue samples. $\mathrm{n}=59$ in each group. (D and E) Kaplan-Meier curves for (D) overall survival or (E) disease-free survival in two groups defined by low and high expression of CSN1 in patients with HCC. (F) CSN1 protein levels in HCC cell lines compared with the normal human liver cell line MIHA. ** P<0.01 and ${ }^{* * *} \mathrm{P}<0.001$. CSN1, constitutive photomorphogenesis 9 signalosome subunit $1 ; \mathrm{HCC}$, hepatocellular carcinoma.

Protein synthesis inhibition assay. CHX or actinomycin D were used to block the endogenous protein synthesis. MHCC-LM3 cells were seeded at $3 \times 10^{5}$ cells/well in a 6-well plate and transfected with the indicated constructs. At $48 \mathrm{~h}$ after transfection, the cells were treated with $0.1 \%$ DMSO (Beijing Solarbio Science \& Technology Co., Ltd.), $75 \mu \mathrm{g} / \mathrm{ml}$ CHX (Sigma-Aldrich; Merck KGaA) and $50 \mu \mathrm{g} / \mathrm{ml}$ actinomycin D (Selleck, Inc.) respectively for $6 \mathrm{~h}$. The cells were then lysed for western blotting. The bands were scanned, and the image semi-quantified using ImageJ software (v1.48; National institutes of Health).

Xenograft growth assay. BALB/cA-nu nude male mice (4-week-old) were obtained from the Animal Laboratory of Daping Hospital, Army Medical University. The mice were maintained under standard animal housing conditions $\left(24^{\circ} \mathrm{C}, 60 \%\right.$ humidity, 12 -h light/dark cycle, free access to food and purified water) and were divided into two groups $\left(\mathrm{n}=5\right.$ each). A total of $5 \times 10^{6}$ MHCC-LM3 cells from CSN1 lentivirus-transfected cells and negative control cells were injected subcutaneously into the mice. At 8 days post-injection, tumor appearance was examined in the mice. The subcutaneous tumor size was calculated and recorded every 4 days using a Vernier caliper. The tumor volume $\left(\mathrm{mm}^{3}\right)$ was calculated as [length $\mathrm{x}$ (width) $\left.{ }^{2}\right] / 2$. At 28 days post-cellular injection, the mice were anesthetized with pentobarbital sodium $(100 \mathrm{mg} / \mathrm{kg}$ body weight) and sacrificed by cervical dislocation. Subsequently, the tumors were excised, and tumor weights were recorded for analysis. All animal experiments were conducted in accordance with the Guide for the Care and Use of Laboratory Animals by the National Institutes of Health. The experimental study design was approved by the Laboratory Animal Welfare and Ethics Committee of the Army Medical University.

Statistical analysis. All statistical analyses were performed using SPSS 20.0 (IBM Corp.) and GraphPad Prism 8.0 (GraphPad Software, Inc.). All results are presented as the mean \pm SD of three experiments. Differences between two groups were analyzed using the unpaired Student's t-test. Differences among $>2$ groups were analyzed using one-way ANOVA followed by Tukey's post hoc test. Wilcoxon signed-rank test (paired data) and Mann-Whitney's U test (unpaired data) were used to analyze the difference in CSN1 expression between HCC and adjacent tissues. Survival analysis was evaluated using the Kaplan-Meier method and the log-rank test. $\mathrm{P}<0.05$ was considered to indicate a statistically significant difference.

\section{Results}

Elevated CSN1 expression indicates poor prognosis in patients with HCC. To characterize CSN1 expression in HCC, CSN1 immunohistochemical staining was conducted on a tissue microarray, which included $117 \mathrm{HCC}$ tissue samples and 58 matched adjacent paracancerous tissue samples. Higher 
Table I. Relationship between the expression of CSN1 and clinicopathological characteristics of patients with hepatocellular carcinoma.

\begin{tabular}{|c|c|c|c|c|}
\hline Clinicopathological characteristics & Cases, n (\%) & CSN1 expression, mean \pm SEM & t-value & P-value \\
\hline \multicolumn{5}{|l|}{ Sex } \\
\hline Male & $106(90.6)$ & $39.12 \pm 2.50$ & 1.414 & 0.160 \\
\hline Female & $11(9.4)$ & $27.52 \pm 8.48$ & & \\
\hline \multicolumn{5}{|l|}{ Age, years } \\
\hline$\geq 50$ & $74(63.3)$ & $36.71 \pm 3.03$ & 0.712 & 0.475 \\
\hline$<50$ & $43(36.7)$ & $40.29 \pm 3.98$ & & \\
\hline \multicolumn{5}{|l|}{ HBsAg } \\
\hline Positive & $106(90.6)$ & $37.57 \pm 2.57$ & 0.591 & 0.556 \\
\hline Negative & $11(9.4)$ & $42.45 \pm 6.45$ & & \\
\hline \multicolumn{5}{|l|}{ AFP, ng/ml } \\
\hline$\geq 400$ & $65(55.6)$ & $40.02 \pm 3.27$ & 0.923 & 0.358 \\
\hline$<400$ & $52(44.4)$ & $35.54 \pm 3.55$ & & \\
\hline \multicolumn{5}{|l|}{ Tumor size, $\mathrm{cm}$} \\
\hline$\geq 5$ & $74(63.2)$ & $42.42 \pm 2.96$ & 2.448 & 0.016 \\
\hline$<5$ & $43(36.8)$ & $30.47 \pm 3.90$ & & \\
\hline \multicolumn{5}{|l|}{ Differentiation } \\
\hline High/moderate & $93(79.5)$ & $37.44 \pm 2.71$ & 0.476 & 0.635 \\
\hline Low & $24(20.5)$ & $40.29 \pm 5.31$ & & \\
\hline \multicolumn{5}{|l|}{ TNM stage ${ }^{\mathrm{a}}$} \\
\hline $\mathrm{I} / \mathrm{II}$ & $64(54.7)$ & $31.21 \pm 3.33$ & 3.241 & 0.002 \\
\hline III/IV & $53(45.3)$ & $46.26 \pm 3.15$ & & \\
\hline \multicolumn{5}{|l|}{ BCLC stage $^{\mathrm{b}}$} \\
\hline $\mathrm{A} / \mathrm{B}$ & $63(53.8)$ & $30.83 \pm 3.36$ & 3.373 & 0.001 \\
\hline $\mathrm{C} / \mathrm{D}$ & $54(46.2)$ & $46.43 \pm 3.10$ & & \\
\hline \multicolumn{5}{|l|}{ Vascular invasion } \\
\hline Yes & $38(32.5)$ & $46.95 \pm 3.60$ & 2.636 & 0.010 \\
\hline No & $79(67.5)$ & $33.74 \pm 3.01$ & & \\
\hline \multicolumn{5}{|l|}{ Intrahepatic metastasis } \\
\hline Yes & $40(34.2)$ & $46.25 \pm 3.86$ & 2.519 & 0.013 \\
\hline No & $77(65.8)$ & $33.76 \pm 2.96$ & & \\
\hline \multicolumn{5}{|l|}{ Lymph node metastasis } \\
\hline Yes & $12(10.3)$ & $54.00 \pm 7.96$ & 2.285 & 0.024 \\
\hline No & $105(89.7)$ & $36.20 \pm 2.47$ & & \\
\hline
\end{tabular}

${ }^{a}$ TNM staging was based on the 8th Edition of The American Joint Committee on Cancer Staging Manual. ${ }^{\mathrm{b} B C L C}$ staging was based on the Edition of the Barcelona-Clinic Liver Cancer Group in 1999. HBsAg, hepatitis B surface antigen; AFP, $\alpha$-fetoprotein.

levels of CSN1 were observed in HCC tissue, compared with the matched adjacent tissues (Fig. 1A-C). Subsequently, the effects of CSN1 expression on the pathological characteristics and prognosis of patients with HCC were analyzed. CSN1 expression levels correlated with tumor size, lymph node metastasis, vascular invasion, intrahepatic metastasis and tumor stage (Table I). Moreover, the overall survival (17.38 vs. $37.78 \% ; \mathrm{P}=0.0285)$ and the disease-free survival rates $(11.30$ vs. $25.74 \%$; $\mathrm{P}=0.0281$ ) of HCC patients with high CSN1 expression were significantly lower compared with patients with low expression (Fig. 1D and E). In addition, CSN1 expression was significantly higher in the HCC cell lines MHCC-LM3,
Hep3B and MHCC-97H compared with the normal liver cell line MIHA (Fig. 1F). These results demonstrated that elevated CSN1 levels were associated with poor prognosis.

CSN1 knockdown inhibits HCC cell proliferation, migration and invasion. To investigate the role of CSN1 in HCC cells, three siRNA candidates were designed and transfected into MHCC-LM3, Hep3B and MHCC-97H cells, and the most efficient siRNA was screened for using western blotting (Fig. 2A). Subsequently, functional analyses were conducted in MHCC-LM3 and Hep3B cells following CSN1 siRNA knockdown. CSN1 knockdown significantly reduced cell 
A

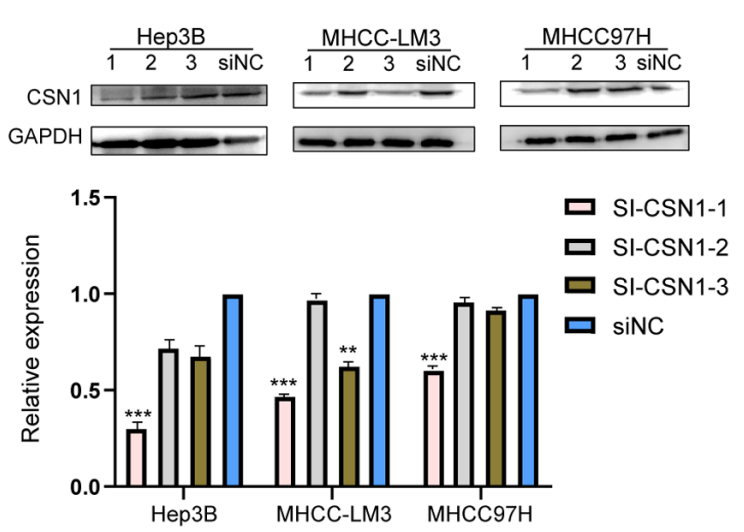

C

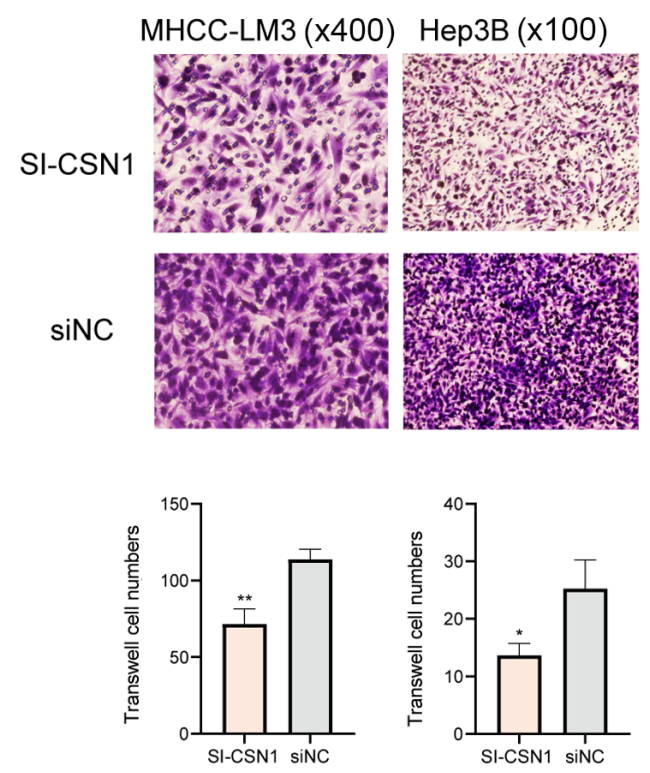

E
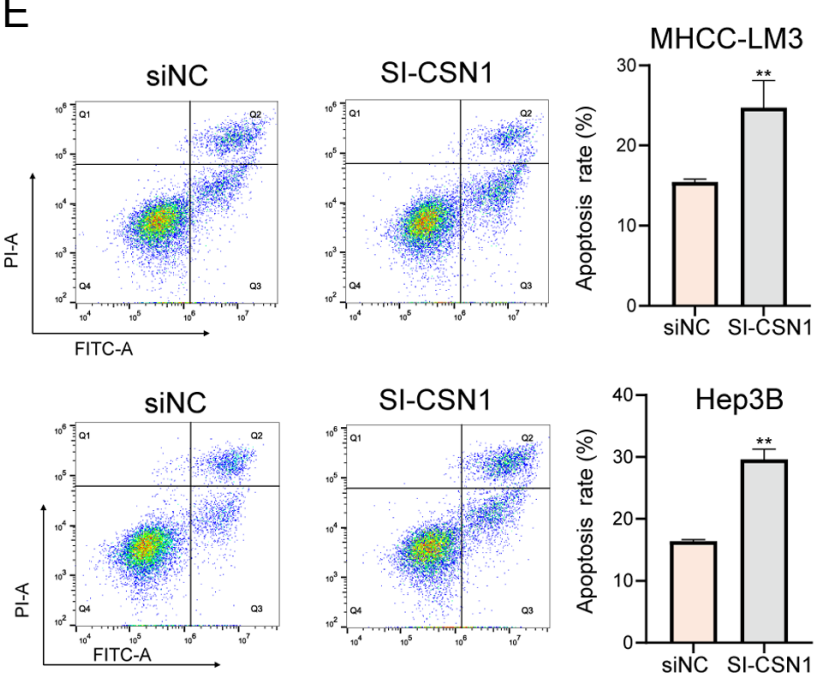

B
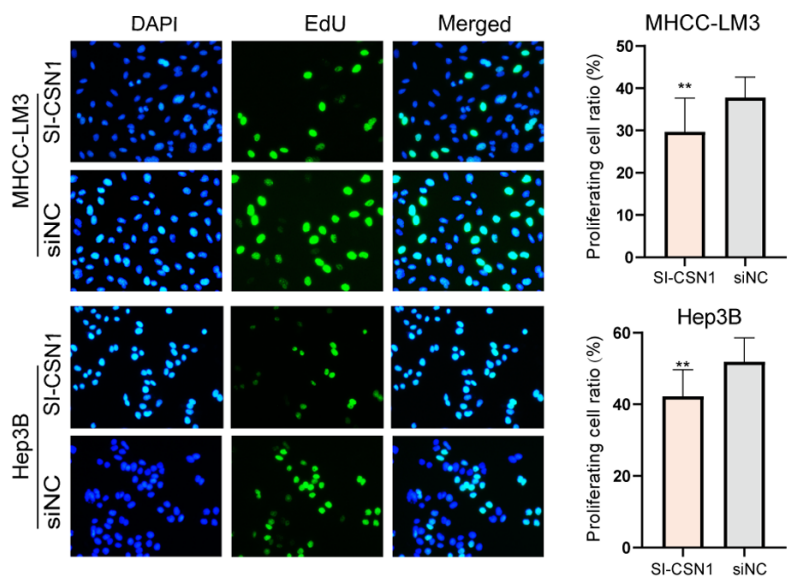

D
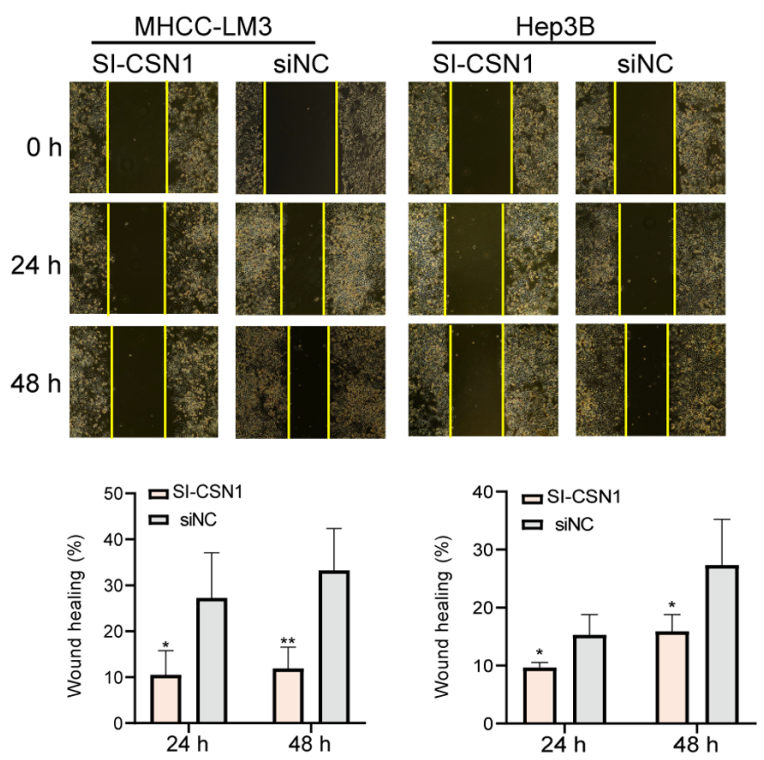

F
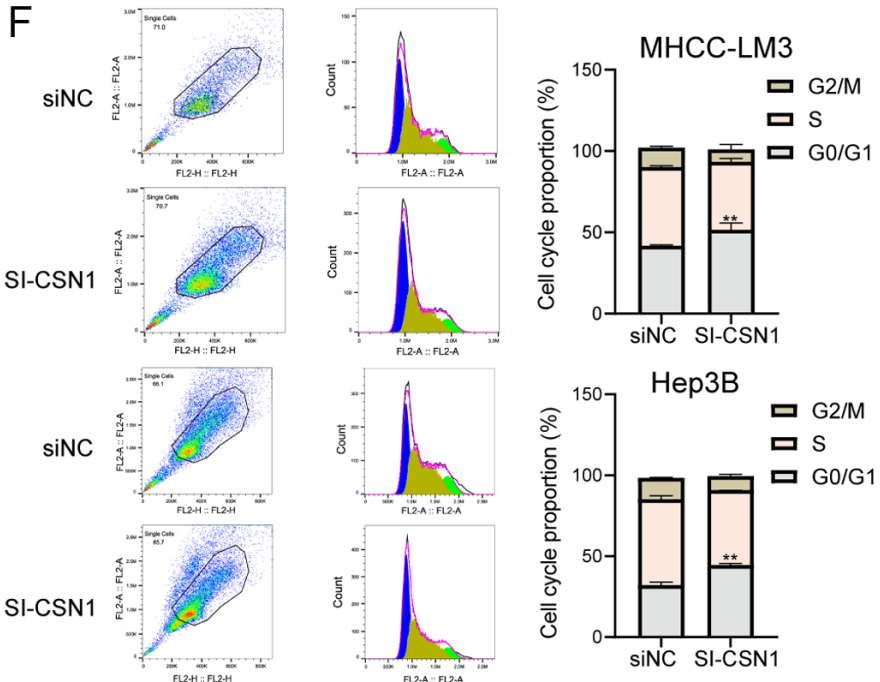

Figure 2. Effects of CSN1 on hepatocellular carcinoma cell proliferation, cell cycle and apoptosis. (A) CSN1 expression levels in MHCC-LM3, Hep3B and MHCC-97H cells transfected with si-CSN1-1, -2 and -3 or siNC. (B) Proliferation of MHCC-LM3 and Hep3B cells following CSN1 knockdown. Magnification, x200. (C) Representative images (upper panel) and quantification (lower panel) of the Transwell assays for MHCC-LM3 and Hep3B cells transfected with si-CSN1 or si-NC. Magnifications, x100 and x400. (D) Measurement of the migration rate after CSN1 knockdown in MHCC-LM3 and Hep3B cells. Magnification, $\mathrm{x} 100$. (E) Apoptosis and (F) cell cycle measurement following CSN1 silencing in MHCC-LM3 and Hep3B cells. ${ }^{*} \mathrm{P}<0.05$, ${ }^{* *} \mathrm{P}<0.01$ and ${ }^{* * * *} \mathrm{P}<0.001$. CSN1, constitutive photomorphogenesis 9 signalosome subunit 1 ; si, small interfering RNA; NC, negative control.

proliferation, compared with NC (Fig. 2B). Moreover, cell migration also significantly decreased after CSN1 knockdown (Fig. 2C and D). Lastly, CSN1 knockdown increased the apoptosis rate (Fig. 2E) and affected cell cycle distribution. The proportion of cells in the $G_{0} / G_{1}$ phase significantly increased, while the proportion of cells in $S$ and $\mathrm{G}_{2} / \mathrm{M}$ phases 
A

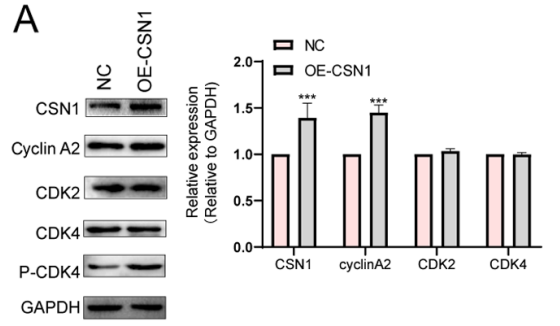

C

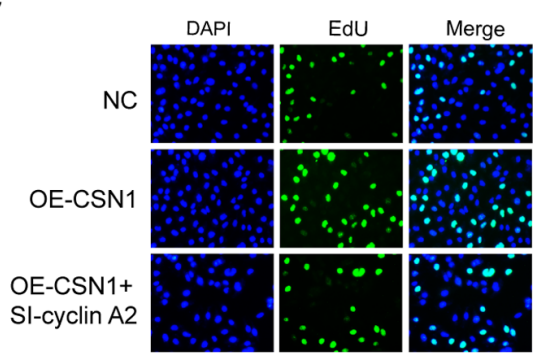

E

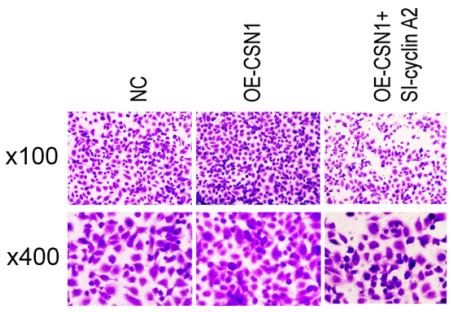

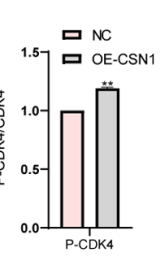

B
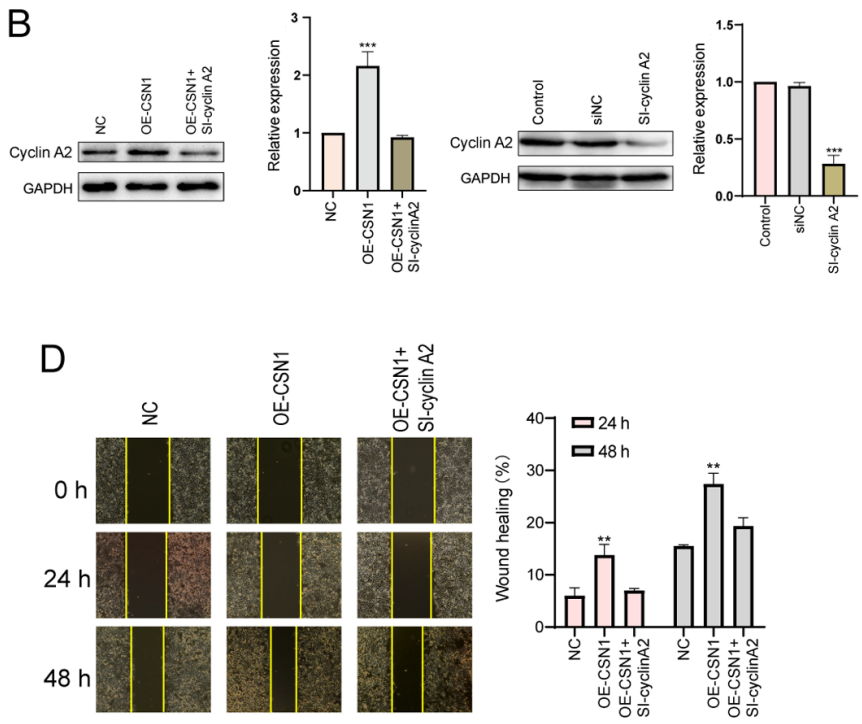

$\mathrm{F}$

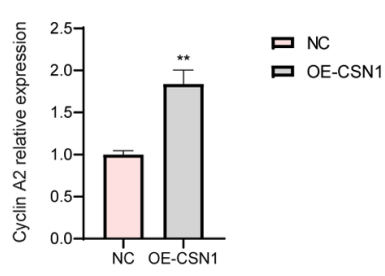

$\mathrm{H}$
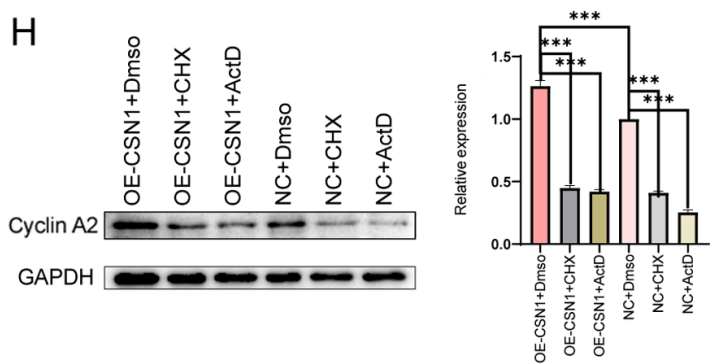

Figure 3. CSN1 interacts with cyclin A2. (A) Representative western blots of CSN1, cyclin A2 and cell cycle-related proteins following CSN1 overexpression in MHCC-LM3 cells. Cells transfected with empty vectors were used as NC group. (B) Western blots of cyclin A2 protein levels in MHCC-LM3 cells transfected with NC or OE-CSN1 plasmids, with or without si-cyclin A2. (C) Proliferation (magnification, x200), (D) wound healing (magnification, x100) (E) and Transwell assay (magnification, x100 and x400) of MHCC-LM3 cells transfected with NC or OE-CSN1 plasmids, with or without si-cyclin A2. (F) Effect of OE-CSN1 on cyclin A2 mRNA levels. (G) Representative western blots of cyclin A2 turnover rate in OE-CSN1 MHCC-LM3 cells following CXH treatment. (H) Cyclin A2 protein levels after OE-CSN1 transfection, and CHX or ActD treatment in MHCC-LM3 cells. ${ }^{*} \mathrm{P}<0.05$, ${ }^{* *} \mathrm{P}<0.01$ and ${ }^{* * * *} \mathrm{P}<0.001$. CHX, cycloheximide; CSN1, constitutive photomorphogenesis 9 signalosome subunit 1; OE, overexpression; ActD, actinomycin D; CDK, cyclin-dependent kinase; si, small interfering; NC, negative control.

significantly decreased after CSN1 knockdown (Fig. 2F). In conclusion, CSN1 knockdown inhibited HCC cell proliferation, migration and invasion.

CSN1 affects HCC cell proliferation and migration via regulating cyclin $A 2$ expression. Cyclin A2 is reported to regulate tumor growth and metastasis $(34,35)$. To investigate the mechanisms by which CSN1 affects HCC proliferation, the expression levels of several cell cycle-related proteins were assessed following CSN1 overexpression. Cyclin A2 and p-CDK4 expressions were upregulated by CSN1 overexpression (Fig. 3A).

Moreover, co-transfection of si-cyclin A2 and OE-CSN1 were carried out. The efficiency of cyclin A2 siRNA transfections was confirmed by western blotting (Fig. 3B). Co-transfection of si-cyclin A2 with OE-CSN1 significantly reduced the effects of OE-CSN1 on cyclin A2 levels, proliferation and migration, compared with cells transduced with OE-CSN1 alone. Thus, cyclin A2 may be a downstream regulator of CSN1 (Fig. 3B-E).

Since CSN subunits may inhibit the ubiquitination and degradation of several protein substrates (22), the effect of CSN1 on cyclin A2 degradation was evaluated. Cyclin A2 mRNA levels were elevated by CSN1 overexpression (Fig. 3F), and the half-life of cyclin A2 protein was not affected by CSN1 overexpression (Fig. 3G). Moreover, inhibition of mRNA transcription or translation by actinomycin D and cycloheximide, respectively, reduced cyclin A2 protein levels 
A

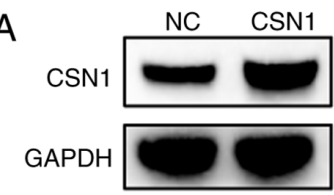

C

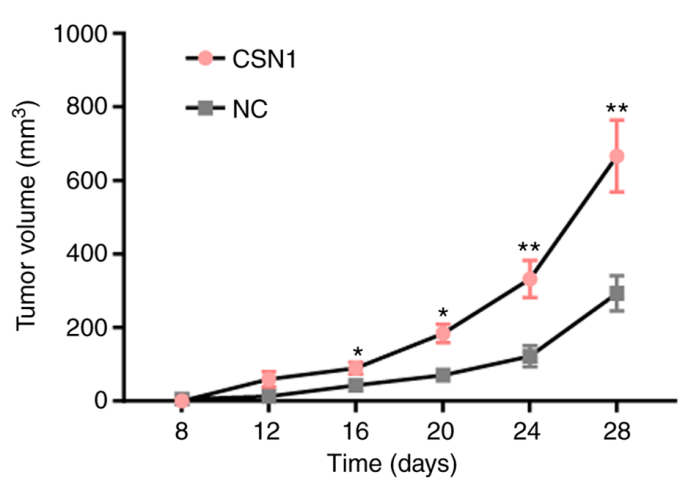

B
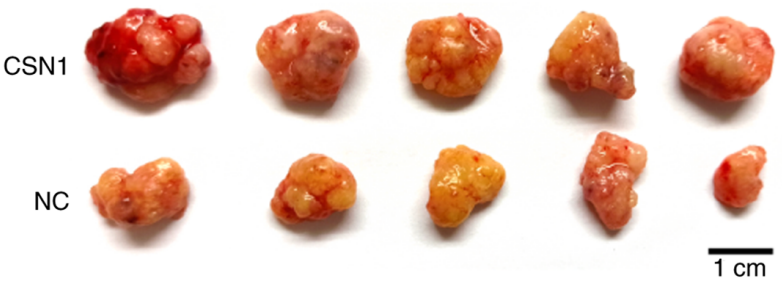

D

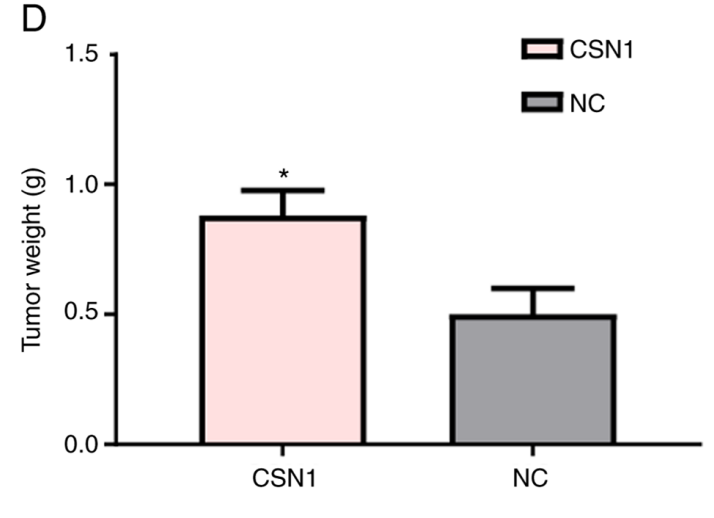

Figure 4. Effects of CSN1 on tumor growth in vivo. Nude mice were subcutaneously injected with CSN1-overexpressing MHCC-LM3 cells and NC cells. Tumor sizes were measured after sizeable tumor formation (day 8). On day 28, the tumors were excised for analysis. $\mathrm{n}=5$. (A) Western blot images of CSN1 levels. (B) Macroscopic appearance of isolated tumors. (C) Tumor volume and (D) weights in CSN1 and NC xenograft nude mice. ${ }^{*}<<0.05$ and ${ }^{* *} \mathrm{P}<0.01$. CSN1, constitutive photomorphogenesis 9 signalosome subunit 1 ; NC, negative control.

in CSN1-overexpressing cells (Fig. 3H), indicating that CSN1 may regulate cyclin $\mathrm{A} 2$ expression at the translational level or in an indirect manner. In conclusion, these results indicated that CSN1 promoted HCC cell proliferation and migration by upregulating cyclin A2 expression.

CSN1 facilitates HCC growth in vivo. To further determine the effects of CSN1 on the tumor growth of HCC cells in vivo, a xenograft tumor assay was performed with stably infected OE-CSN1 and NC MHCC-LM3 cells (Fig. 4A). CSN1 overexpression significantly increased the tumor volumes and weights of the MHCC-LM3 cells compared with the negative control group (Fig. 4B). Based on the tumor volumes, CSN1 overexpression was associated with higher tumor growth rate and tumor weights (Fig. 4C and D). These results suggested that CSN1 overexpression can facilitate tumor growth in vivo.

\section{Discussion}

Hepatocellular carcinoma (HCC) is the most common primary malignant tumor of the liver, accounting for $\sim 85 \%$ of all cases (36). The high mortality rate of liver cancer is mainly associated with tumor invasion, metastasis and tumor recurrence after surgical resection $(6,37)$. In recent years, a number of studies have confirmed that the CSN superfamily is widely involved in the regulation of several important intracellular pathways, including cell proliferation, migration, invasion and signal transduction, and plays an important role in tumor development (13,38-40). For example, CSN5 and CSN6 are highly expressed in myeloma, lung, colon, breast cancer, malignant glioma and leukemia clinical tissues (14). However, the expression pattern and role of CSN1 in HCC have yet to be determined.
The present study reported that CSN1 expression levels were increased in HCC tissues and HCC cells compared to normal controls. Of note, because of the tumor heterogeneity, $30.5 \%$ of the HCC tissues displayed a decrease or no difference in CSN1 expression compared to adjacent tissues. High CSN1 levels indicated poor prognosis in patients. In addition, in vitro assays indicated that CSN1 knockdown inhibited the proliferation and migration of HCC cells, suggesting that CSN1 may promote the occurrence and development of HCC by enhancing proliferation and inhibiting apoptosis. Moreover, the xenograft growth assay revealed that CSN1 facilitated HCC growth in vivo. Mechanically, CSN1 can affect the expression of cyclin A2 and thus participate in the regulation of the cell cycle, cell proliferation and migration. Cyclin A2 is a key regulator of cell cycle transition and can regulate cell growth through its pro-mitotic effects. Cyclin A2 modulates epithelial-mesenchymal transition via $\beta$-catenin and phospholipase C pathways (41). The present study supported the notion that CSN1 played a role in carcinogenesis and cancer progression.

The role of the CSN complex in ubiquitin-mediated protein degradation is well-characterized. Several CSN subunits exert de-ubiquitination activity. For example, CSN5 promoted the proliferation of non-small cell lung cancer cells by inhibiting the ubiquitination-mediated degradation of survivin (17). Du et al (42) demonstrated that CSN6 promoted the occurrence of gastric cancer via the ubiquitin-independent proteasomal degradation of p16INK4a. In addition, CSN is also involved in ubiquitin-like modifications. For example, the CSN complex de-neddylates cullins and destabilizes E3 ligase complexes (43). De-neddylation by the CSN complex inactivates a cullin 1 complex that ubiquitinates capicua following its phosphorylation by MAP kinase in response to EGFR 
signaling (44). The CUL4A-RBX1-DDB1-DDB2 complex $\left(C R L 4 A^{\mathrm{DDB} 2}\right)$ is inactive in the absence of damaged DNA and requires CSN to regulate the repair process (45).

However, the present results indicated that although CSN1 can increase the expression of cyclin A2 mRNA, the regulatory effects of CSN1 on cyclin A2 may not occur at the post-translational level, since the protein half-time of cyclin A2 was not affected by CSN1. There are several possible reasons for this unexpected result. First, CSN1 may not interact with cyclin A2 directly, and there may be other mediators involved in the CSN1-cyclin A2 axis. Secondly, cyclin A2 may not be a direct substrate in CSN-mediated ubiquitination. Moreover, CSN1 might not be the subunit in the CSN complex that mediates the ubiquitination of its substrates. It has been suggested that the MPN domain in CSN6 and CSN5 may be responsible for regulating ubiquitination and cullin de-neddylation $(14,46,47)$. Since CSN1 lacks an MPN domain, there is still no evidence that CSN1 is directly involved in ubiquitination.

There are several limitations in the present study. The exact molecular mechanism of CSN1 in HCC tumorigenesis needs further investigation, especially regarding how CSN1 affects cyclin A2 to regulate HCC cell proliferation and migration. Moreover, how CSN1 and other CSN components interplay to regulate HCC tumorigenesis should also be evaluated in the future.

Taken together, the present findings indicated that high expression levels of CSN1 may contribute to HCC progression. CSN1 knockdown inhibited HCC cell proliferation and induced apoptosis and migration by affecting cyclin A2 expression. The present study determined the function of CSN1 and may provide new insights and therapeutic strategies for HCC prevention and treatment.

\section{Acknowledgements}

The authors would like to thank the Hepatobiliary Surgery laboratory of Xinan Hospital, Army Medical University for providing laboratory resources.

\section{Funding}

The present study was supported by the National Natural Science Foundation of China (grant no. 81270523).

\section{Availability of data and materials}

The datasets used and/or analyzed during the current study are available from the corresponding author on reasonable request.

\section{Authors' contributions}

PC and JC designed the experiments. HF and YZ performed most of the experiments and drafted the manuscript. YC conducted the experiments on the clinical samples. All authors read and approved the final manuscript.

\section{Ethics approval and consent to participate}

Informed consent was obtained from all participants. Experiments involving clinical samples conformed to the principles of the Declaration of Helsinki and were approved by The Ethics Committee of Daping Hospital, Army Medical University. All procedures involving mice and the corresponding experimental protocols were approved by The Laboratory Animal Welfare and Ethics Committee of the Army Medical University.

\section{Patient consent for publication}

Not applicable.

\section{Competing interests}

The authors declare that they have no competing interests.

\section{References}

1. Bray F, Ferlay J, Soerjomataram I, Siegel RL, Torre LA and Jemal A: Global cancer statistics 2018: GLOBOCAN estimates of incidence and mortality worldwide for 36 cancers in 185 countries. CA Cancer J Clin 68: 394-424, 2018.

2. Chen W, Zheng R, Baade PD, Zhang S, Zeng H, Bray F, Jemal A, Yu XQ and He J: Cancer statistics in China, 2015. CA Cancer J Clin 66: 115-132, 2016.

3. Juarez-Hernandez E, Motola-Kuba D, Chavez-Tapia NC, Uribe $\mathrm{M}$ and Barbero Becerra V: Biomarkers in hepatocellular carcinoma: An overview. Expert Rev Gastroenterol Hepatol 11: 549-558, 2017.

4. Yan X, Shao C, Chen C, Chen J, Gu S, Huang L, Fu X, Zhao H and Qiu Y: Mutation detection of fibroblast growth factor receptor 3 for infiltrative hepatocellular carcinoma by whole-exome sequencing. Dig Dis Sci 62: 407-417, 2017.

5. Zhu Q, Li N, Zeng X, Han Q, Li F, Yang C, Lv Y, Zhou Z and Liu Z: Hepatocellular carcinoma in a large medical center of China over a 10-year period: Evolving therapeutic option and improving survival. Oncotarget 6: 4440-4450, 2015.

6. Kumari R, Sahu MK, Tripathy A, Uthansingh K and Behera M: Hepatocellular carcinoma treatment: Hurdles, advances and prospects. Hepatic Oncol 5: Hep08, 2018.

7. Heinrich B, Czauderna C and Marquardt JU: Immunotherapy of hepatocellular carcinoma. Oncol Res Treat 41: 292-297, 2018

8. Chamovitz DA, Wei N, Osterlund MT, von Arnim AG, Staub JM, Matsui $M$ and Deng XW: The COP9 complex, a novel multisubunit nuclear regulator involved in light control of a plant developmental switch. Cell 86: 115-121, 1996.

9. Wei N and Deng XW: COP9: A new genetic locus involved in light-regulated development and gene expression in arabidopsis. Plant Cell 4: 1507-1518, 1992.

10. Wei N and Deng XW: Making sense of the COP9 signalosome. A regulatory protein complex conserved from Arabidopsis to human. Trends Genet 15: 98-103, 1999.

11. Wang H, Ma LG, Li JM, Zhao HY and Deng XW: Direct interaction of Arabidopsis cryptochromes with COP1 in light control development. Science 294: 154-158, 2001.

12. Schwechheimer C and Deng XW: The COP/DET/FUS proteins-regulators of eukaryotic growth and development. Semin Cell Dev Biol 11: 495-503, 2000.

13. Richardson KS and Zundel W: The emerging role of the COP9 signalosome in cancer. Mol Cancer Res 3: 645-653, 2005.

14. Lee MH, Zhao R, Phan L and Yeung SC: Roles of COP9 signalosome in cancer. Cell Cycle 10: 3057-3066, 2011.

15. Wei N and Deng XW: The COP9 Signalosome. Annu Rev Cell Dev Biol 19: 261-286, 2003.

16. Gusmaroli G, Figueroa P, Serino G and Deng XW: Role of the MPN subunits in COP9 signalosome assembly and activity, and their regulatory interaction with Arabidopsis Cullin3-based E3 ligases. Plant Cell 19: 564-581, 2007.

17. Li J, Li Y, Wang B, Ma Y and Chen P: CSN5/Jab1 facilitates non-small cell lung cancer cell growth through stabilizing survivin. Biochem Biophys Res Commun 500: 132-138, 2018.

18. Liu H, Hu J, Pan H, Luo D, Huang M and Xu W: CSN5 promotes hepatocellular carcinoma progression by SCARA5 inhibition through suppressing $\beta$-catenin ubiquitination. Dig Dis Sci 63: $155-165,2018$. 
19. Mao Z, Sang MM, Chen C, Zhu WT, Gong YS and Pei DS: CSN6 promotes the migration and invasion of cervical cancer cells by inhibiting autophagic degradation of cathepsin L. Int J Biol Sci 15: 1310-1324, 2019.

20. Shi J, Guan X, Zhan F, Liu C, Li Z, Yao Y, Wang B, Lou C and Zhang Y: CSN6 expression is associated with pancreatic cancer progression and predicts poor prognosis. Cancer Biol Ther 20: $1290-1299,2019$.

21. Suisse A, Bekes M, Huang TT and Treisman JE: The COP9 signalosome inhibits Cullin-RING E3 ubiquitin ligases independently of its deneddylase activity. Fly (Austin) 12: 118-126, 2018.

22. Schwechheimer C and Deng XW: COP9 signalosome revisited: A novel mediator of protein degradation. Trends Cell Biol 11: 420-426, 2001.

23. Bech-Otschir D, Seeger M and Dubiel W: The COP9 signalosome: At the interface between signal transduction and ubiquitin-dependent proteolysis. J Cell Sci 115: 467-473, 2002.

24. Rhodes DR, Kalyana-Sundaram S, Mahavisno V, Varambally R, Yu J, Briggs BB, Barrette TR, Anstet MJ, Kincead-Beal C, Kulkarni P, et al: Oncomine 3.0: Genes, pathways, and networks in a collection of 18,000 cancer gene expression profiles. Neoplasia 9: 166-180, 2007.

25. Rhodes DR, Yu J, Shanker K, Deshpande N, Varambally R, Ghosh D, Barrette T, Pandey A and Chinnaiyan AM: ONCOMINE: A cancer microarray database and integrated data-mining platform. Neoplasia 6: 1-6, 2004.

26. Xu M, Zhen L, Lin L, Wu K, Wang Y and Cai X: Overexpression of CSN6 promotes the epithelial-mesenchymal transition and predicts poor prognosis in hepatocellular carcinoma. Clin Res Hepatol Gastroenterol 44: 340-348, 2019.

27. Yu YS, Tang ZH, Pan QC, Chen XH, Liu XN and Zang GQ Inhibition of Csn3 expression induces growth arrest and apoptosis of hepatocellular carcinoma cells. Cancer Chemother Pharmacol 69: 1173-1180, 2012.

28. Deng H, Cheng Y, Guo Z, Zhang F, Lu X, Feng L, Wang X and Xu Z: Overexpression of CyclinA2 ameliorates hypoxia-impaired proliferation of cardiomyocytes. Exp Ther Med 8: 1513-1517, 2014.

29. Gopinathan L, Tan SL, Padmakumar VC, Coppola V, Tessarollo L and Kaldis P: Loss of Cdk2 and cyclin A2 impairs cell proliferation and tumorigenesis. Cancer Res 74: 3870-3879, 2014.

30. Yasmeen A, Berdel WE, Serve H and Müller-Tidow C: E- and A-type cyclins as markers for cancer diagnosis and prognosis. Expert Rev Mol Diagn 3: 617-633, 2003.

31. Ohashi R, Gao C, Miyazaki M, Hamazaki K, Tsuji T, Inoue Y, Uemura T, Hirai R, Shimizu $N$ and Namba M: Enhanced expression of cyclin $\mathrm{E}$ and cyclin $\mathrm{A}$ in human hepatocellular carcinomas. Anticancer Res 21: 657-662, 2001.

32. Livak KJ and Schmittgen TD: Analysis of relative gene expression data using real-time quantitative PCR and the 2(-Delta Delta C(T)) method. Methods 25: 402-408, 2001.

33. Fu H, Zhang Y, Chen J, Zhou B, Chen G and Chen P: Tmub1 suppresses hepatocellular carcinoma by promoting the Ubiquitination of $\Delta \mathrm{Np} 63$ isoforms. Mol Ther Oncolytics 18 $126-136,2020$
34. Blanchard JM: Cyclin A2 transcriptional regulation: Modulation of cell cycle control at the G1/S transition by peripheral cues. Biochem Pharmacol 60: 1179-1184, 2000.

35. Bendris N, Cheung CT, Leong HS, Lewis JD, Chambers AF, Blanchard JM and Lemmers B: Cyclin A2, a novel regulator of EMT. Cell Mol Life Sci 71: 4881-4894, 2014.

36. Neureiter D, Stintzing S, Kiesslich T and Ocker M: Hepatocellular carcinoma: Therapeutic advances in signaling, epigenetic and immune targets. World J Gastroenterol 25: 3136-3150, 2019.

37. Chen CP: Role of radiotherapy in the treatment of hepatocellular carcinoma. J Clin Transl Hepatol 7: 183-190, 2019.

38. Wicker CA and Izumi T: Analysis of RNA expression of normal and cancer tissues reveals high correlation of COP9 gene expression with respiratory chain complex components. BMC Genomics 17: 983, 2016.

39. Wang L, Zheng JN and Pei DS: The emerging roles of Jab1/CSN5 in cancer. Med Oncol 33: 90, 2016.

40. Mao Z, Chen C and Pei DS: The emerging role of CSN6 in biological behavior and cancer progress. Anticancer Agents Med Chem 19: 1198-1204, 2019.

41. Cheung CT, Bendris N, Paul C, Hamieh A, Anouar Y, Hahne M, Blanchard JM and Lemmers B: Cyclin A2 modulates EMT via $\beta$-catenin and phospholipase $\mathrm{C}$ pathways. Carcinogenesis 36 : 914-924, 2015.

42. Du W, Liu Z, Zhu W, Li T, Zhu Z, Wei L, Song J and Pei D: CSN6 promotes tumorigenesis of gastric cancer by ubiquitinindependent proteasomal degradation of $\mathrm{p} 16^{\mathrm{INK} 4 \mathrm{a}}$. Cancer Biol Med 16: 514-529, 2019.

43. Schwechheimer C: NEDD8-its role in the regulation of Cullin-RING ligases. Curr Opin Plant Biol 45: 112-119, 2018.

44. Su H, Huang W and Wang X: The COP9 signalosome negatively regulates proteasome proteolytic function and is essential to transcription. Int J Biochem Cell Biol 41: 615-624, 2009.

45. Cavadini S, Fischer ES, Bunker RD, Potenza A, Lingaraju GM, Goldie KN, Mohamed WI, Faty M, Petzold G, Beckwith RE, et al: Cullin-RING ubiquitin E3 ligase regulation by the COP9 signalosome. Nature 531: 598-603, 2016.

46. Lim SO, Li CW, Xia W, Cha JH, Chan LC, Wu Y, Chang SS, Lin WC, Hsu JM, Hsu YH, et al: Deubiquitination and stabilization of PD-L1 by CSN5. Cancer Cell 30: 925-939, 2016.

47. Hou J, Deng Q, Zhou J, Zou J, Zhang Y, Tan P, Zhang W and Cui H: CSN6 controls the proliferation and metastasis of glioblastoma by CHIP-mediated degradation of EGFR. Oncogene 36: 1134-1144, 2017.

This work is licensed under a Creative Commons Attribution-NonCommercial-NoDerivatives 4.0 International (CC BY-NC-ND 4.0) License. 